\title{
1 CNTNAP2 is a differentially expressed gene in human metastatic breast cancer, in the brain and in the lymph nodes.
}

Shahan Mamoor, MS ${ }^{1}$

${ }^{1}$ shahanmamoor@gmail.com

East Islip, NY USA

Metastasis to the brain is a clinical problem in patients with breast cancer ${ }^{1-3}$. We mined published microarray data ${ }^{4,5}$ to compare primary and metastatic tumor transcriptomes for the discovery of genes associated with brain metastasis in humans with metastatic breast cancer. We found that contactin-associated protein-like 2, encoded by CNTNAP2, was among the genes whose expression was most different in the brain and lymph node metastases of patients with metastatic breast cancer. CNTNAP2 mRNA was present at increased quantities in brain metastatic tissues as compared to primary tumors of the breast. Importantly, expression of CNTNAP2 in primary tumors was significantly correlated with patient recurrence-free survival in patients with breast cancer. Modulation of CNTNAP2 expression may be relevant to the biology by which tumor cells metastasize from the breast to the brain while evading immune clearance in the lymph nodes in humans with metastatic breast cancer. These data are one piece of evidence suggesting a common ancestor or tumor clone for brain and lymph node metastases that originate from the primary tumor, alluding to patterns in developmental origin and migratory pathways through the lymph node in human brain metastatic breast cancer.

$\underline{\text { Keywords: }}$ breast cancer, metastasis, brain metastases, central nervous system metastases, lymph node metastases, contactin-associated protein-like 2, CNTNAP2, systems biology of breast cancer, targeted therapeutics in breast cancer. 
One report described a $34 \%$ incidence of central nervous system metastases in patients

\section{Methods}

We used datasets GSE10893 ${ }^{4}$ and GSE124648 5 for this global differential gene expression analysis of brain metastatic breast cancer in conjunction with GEO2R. GSE10893 was generated using Agilent-011521 Human 1A Microarray G4110A technology with $n=11$ primary breast tumors and $n=3$ brain metastases from patients with breast cancer; analysis was performed using platform GPL885. GSE124648 was generated using Affymetrix Human Genome U133A array technology with $n=10$ normal breast tissues and $n=44$ lymph node metastases from patients with breast cancer; analysis was performed using platform GPL96. The Benjamini and Hochberg method of $p$-value adjustment was used for ranking of differential expression but raw $p$-values were used to assess statistical significance of global differential expression. Log-transformation of data was auto-detected, and the NCBI generated category of platform annotation was used. A statistical test was performed to evaluate whether CNTNAP2 gene expression was significantly different between primary tumors of the breast and brain metastases in humans with breast cancer using a two-tailed t-test. For Kaplan-Meier survival analysis, we used the Kaplan-Meier plotter online tool ${ }^{7}$ for correlation of CNTNAP2 mRNA expression levels in primary tumors of the breast with recurrence-free survival in $n=3951$ breast cancer patients.

\section{$\underline{\text { Results }}$}

We performed global comparative transcriptome analysis of metastatic and primary tumor tissues of patients with metastatic breast, as well as normal tissues of the breast using published microarray data ${ }^{4,5}$ to describe the transcriptional landscape of brain metastasis in human breast cancer in an unbiased fashion and for the discovery of novel therapeutic targets.

\section{CNTNAP2 is differentially expressed in the brain metastases of patients with brain metastatic breast cancer.}

Through blind, systems-level analysis of published microarray data ${ }^{4}$, we identified contactin-associated protein-like 2, encoded by CNTNAP2, as a differentially expressed gene in the brain metastatic tissues of humans with breast cancer (Table 1). When sorting each of the 
genes expressed in brain metastases based on significance of difference as compared to primary tumors of the breast in patients with breast cancer, CNTNAP2 ranked 246 out of 17418 total transcripts (Chart 1), equating to $98.6 \%$ differential expression. Differential expression of CNTNAP2 in the brain metastases of patients with metastatic breast cancer was statistically significant (Chart $1 ; p=7.30 \mathrm{E}-04)$.

\section{CNTNAP2 is differentially expressed in the lymph node metastases of patients with breast cancer.}

To determine whether differential expression of CNTNAP2 in human breast cancer could be validated in a second microarray dataset, and whether this was specific to brain metastases or could be observed in other anatomical locations, we queried a second microarray dataset ${ }^{5}$, here comparing normal breast tissues to lymph node metastases. Again, we identified CNTNAP2 as a differentially expressed gene in the lymph node metastatic tissues of patients with breast cancer (Chart 2). When sorting each of the genes expressed in lymph node metastases based on significance of difference as compared to normal breast tissues, CNTNAP2 ranked 4748 out of 22283 total transcripts (Chart 2), equating to $78.7 \%$ differential expression. Differential expression of CNTNAP2 in the lymph node metastases of patients with metastatic breast cancer approached the level of statistical significance (Chart 2; $p=5.38 \mathrm{E}-03$ ). Thus, differential expression of CNTNAP2, transcriptome-wide, in metastatic tissues of women with metastatic breast cancer was conserved across two independent microarray datasets, in the brain and in the lymph nodes.

\section{CNTNAP2 is expressed at higher levels in the brain metastases of patients with metastatic breast cancer.}

We obtained exact mRNA expression levels for CNTNAP2, in primary tumors of the breast and in brain metastasis of patients with brain metastatic breast cancer to determine direction and statistical significance of change in CNTNAP2 expression in brain metastatic tissues. We observed significantly higher expression of CNTNAP2 in the brain metastases of patients with breast cancer as compared to primary tumors of the breast: CNTNAP2 was expressed at $-0.86 \pm 1.56$ arbitrary units $(\mathrm{AU})$ in primary tumors of the breast, while it was expressed at 2.27 $\pm 0.58 \mathrm{AU}$ in brain metastatic tissues (Figure 1). The difference in CNTNAP2 mRNA levels between primary tumors of the breast and brain metastatic tissues was statistically significant (Figure 1; $p=0.002092$ ).

\section{CNTNAP2 expression is correlated with survival outcomes in human breast cancer.}

We performed Kaplan-Meier survival analysis ${ }^{7}$ in 3951 breast cancer patients in total, to evaluate whether CNTNAP2 tumor expression was correlated with survival outcomes in breast cancer. We observed a correlation between primary tumor expression of CNTNAP2 and recurrence-free survival (RFS) in patients with breast cancer (Figure 2). Patients whose primary tumors expressed low levels of CNTNAP2 possessed median RFS of 55 months, while patients whose tumors expressed high levels of CNTNAP2 possessed median RFS of 43.7 months. This difference in RFS based on CNTNAP2 tumor expression in patients with breast cancer trended towards statistical significance (Figure 2, Chart 3; logrank $p$-value: 0.00091 ; hazard ratio: 1.2 (1.08-1.34)). 
Thus, by mining published microarray data ${ }^{4,5}$ in an unbiased and systematic fashion, we identified contactin-associated protein-like 2 , encoded by CNTNAP2, as among the genes whose expression was most different, transcriptome-wide, in the brain and lymph node metastases of patients with breast cancer, when compared to primary tumors of the breast and to normal breast tissues, respectively; we observed significantly increased expression of CNTNAP2 in brain metastases as compared to primary tumors of the breast. Further, we found a correlation between CNTNAP2 expression and patient survival outcomes, as recurrence-free survival was greater in patients whose primary tumors expressed lower levels of CNTNAP2 as compared to patients whose primary tumors expressed higher levels of CNTNAP2.

\section{Discussion}

We provided evidence here that CNTNAP2 is among the genes whose expression is most different in the brain and lymph node metastases of patients with metastatic breast cancer, that CNTNAP2 mRNA is present at significantly increased quantity in brain metastatic tissues as compared to primary tumors of the breast, and that primary tumor CNTNAP2 expression is significantly correlated with patient survival outcomes in human breast cancer. Evaluation of the effects of genetic depletion of CNTNAP2 in mouse models of metastatic breast cancer on metastasis to the central nervous system is merited. Modulation of CNTNAP2 expression may be relevant to the processes by which breast cancer cells exit the breast, enter the vasculature and/or lymphatics, reside in the lymph nodes, evade immune clearance, breach the blood-brain barrier and colonize the brain. These data are one piece of evidence that allude to a common cell or cell(s) of origin for brain and lymph node metastases in human metastatic breast cancer. 


\section{References}

1. Lin, N.U., Amiri-Kordestani, L., Palmieri, D., Liewehr, D.J. and Steeg, P.S., 2013. CNS metastases in breast cancer: old challenge, new frontiers.

2. Bendell, J.C., Domchek, S.M., Burstein, H.J., Harris, L., Younger, J., Kuter, I., Bunnell, C., Rue, M., Gelman, R. and Winer, E., 2003. Central nervous system metastases in women who receive trastuzumab-based therapy for metastatic breast carcinoma. Cancer, 97(12), pp.2972-2977.

3. Tsukada, Y., Fouad, A., Pickren, J.W. and Lane, W.W., 1983. Central nervous system metastasis from breast carcinoma autopsy study. Cancer, 52(12), pp.2349-2354.

4. Weigman, V.J., Chao, H.H., Shabalin, A.A., He, X., Parker, J.S., Nordgard, S.H., Grushko, T., Huo, D., Nwachukwu, C., Nobel, A. and Kristensen, V.N., 2012. Basal-like Breast cancer DNA copy number losses identify genes involved in genomic instability, response to therapy, and patient survival. Breast cancer research and treatment, 133(3), pp.865-880.

5. Sinn, B.V., Fu, C., Lau, R., Litton, J., Tsai, T.H., Murthy, R., Tam, A., Andreopoulou, E., Gong, Y., Murthy, R. and Gould, R., 2019. SET ER/PR: a robust 18-gene predictor for sensitivity to endocrine therapy for metastatic breast cancer. NPJ breast cancer, 5(1), pp.1-8.

6. Awada, A., Colomer, R., Inoue, K., Bondarenko, I., Badwe, R.A., Demetriou, G., Lee, S.C., Mehta, A.O., Kim, S.B., Bachelot, T. and Goswami, C., 2016. Neratinib plus paclitaxel vs trastuzumab plus paclitaxel in previously untreated metastatic ERBB2-positive breast cancer: the NEfERT-T randomized clinical trial. JAMA oncology, 2(12), pp.1557-1564.

7. Györffy, B., Lanczky, A., Eklund, A.C., Denkert, C., Budczies, J., Li, Q. and Szallasi, Z., 2010. An online survival analysis tool to rapidly assess the effect of 22,277 genes on breast cancer prognosis using microarray data of 1,809 patients. Breast cancer research and treatment, 123(3), pp.725-731. 


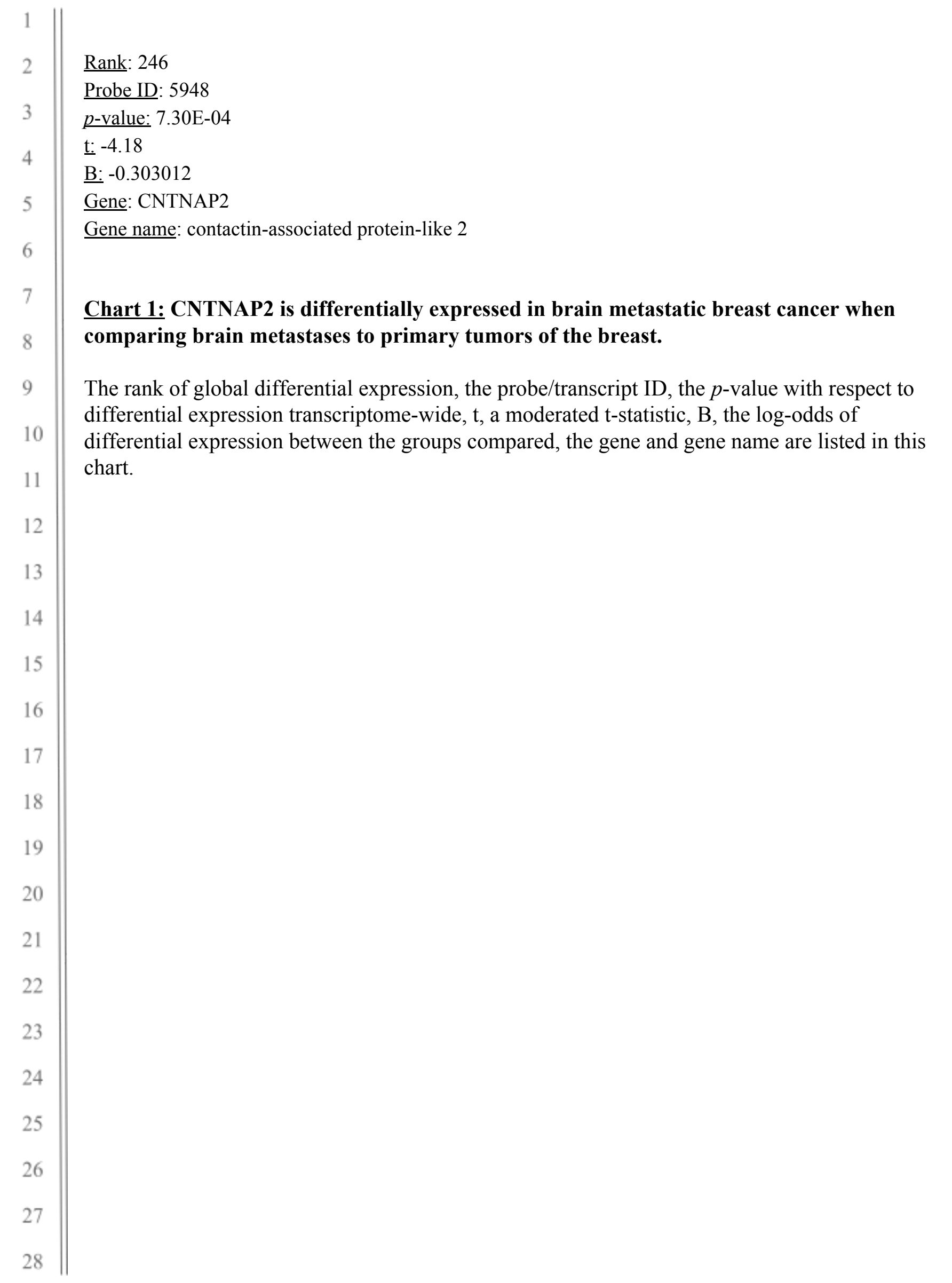




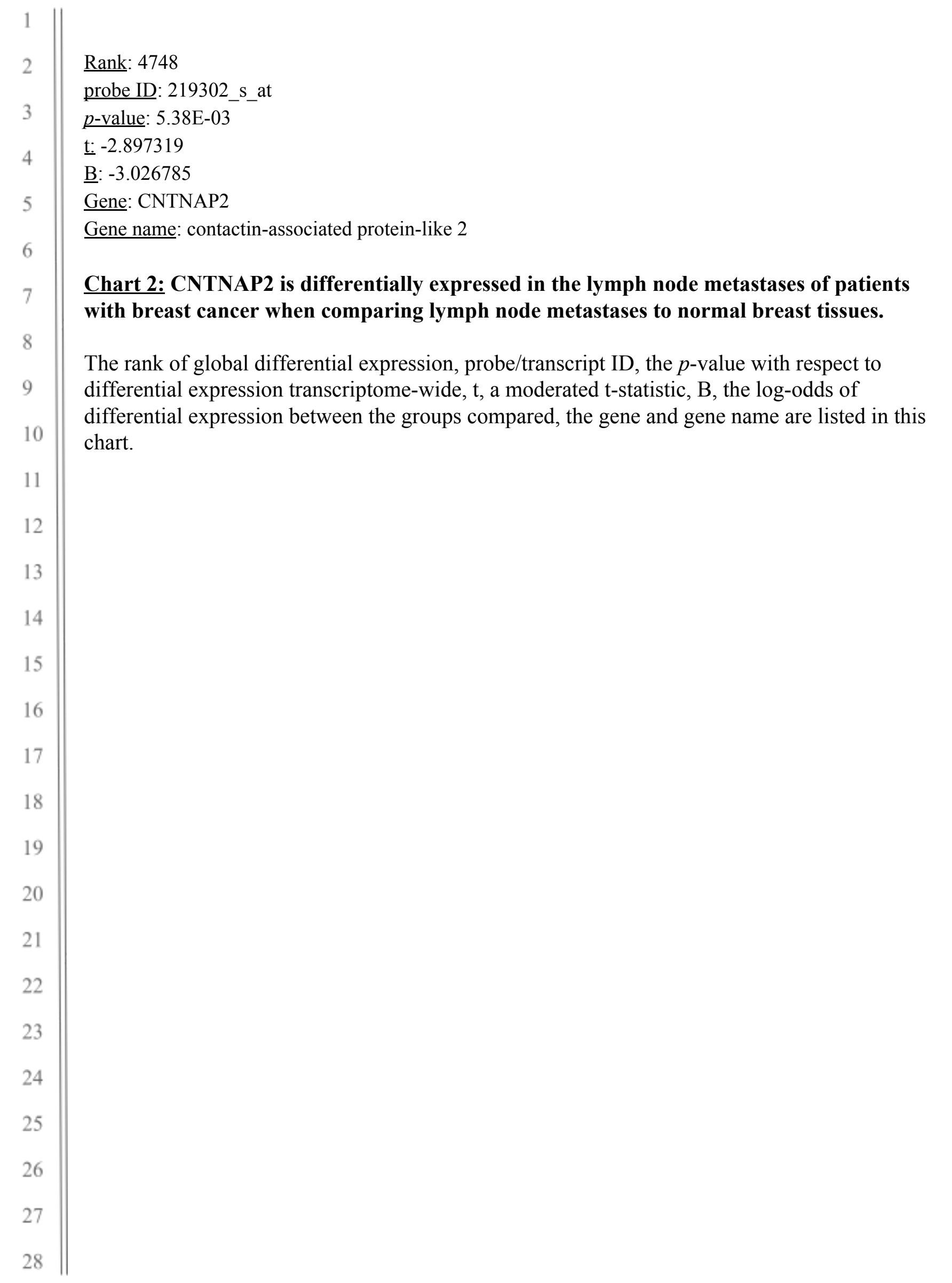




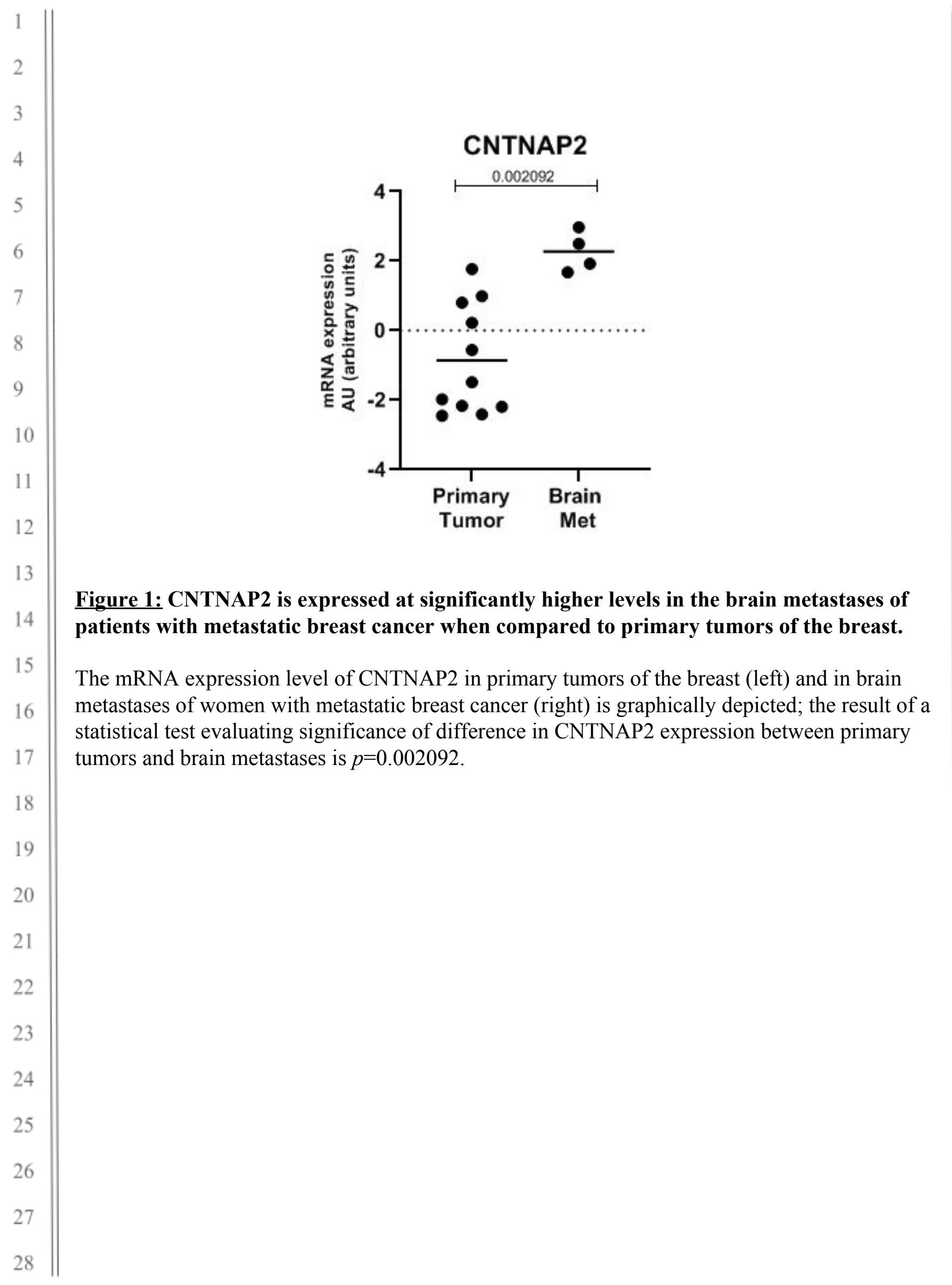




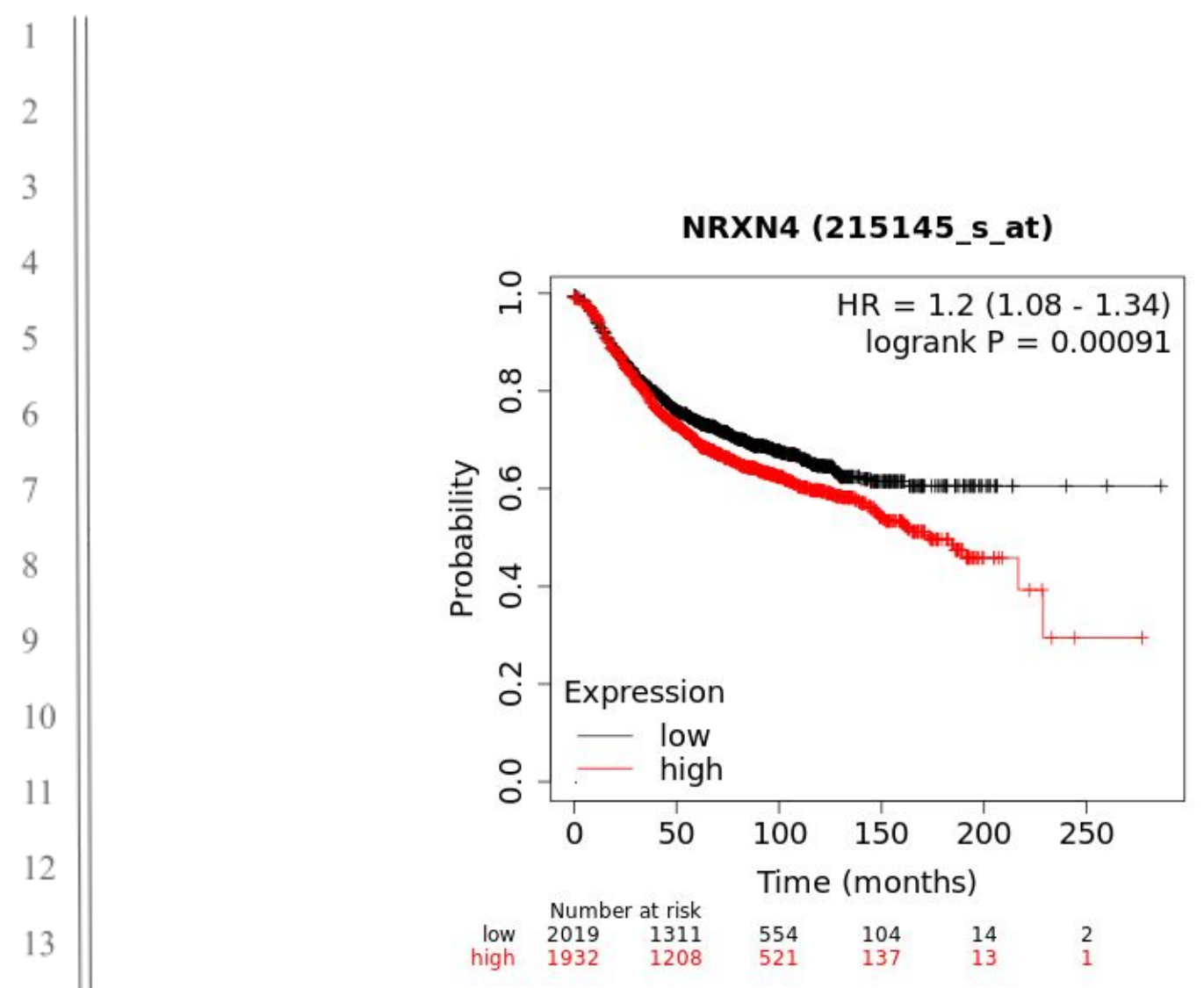

Figure 2: Significant correlation between CNTNAP2 primary tumor expression and recurrence-free survival in patients with breast cancer.

Depicted in this Kaplan-Meier plot is the probability of recurrence-free survival for $n=3951$ total breast cancer patients stratified into two groups, based on low or high expression of CNTNAP2 in patient primary tumors. The log rank $p$-value denoting statistical significance of difference in recurrence-free survival when comparing the two groups, as well as hazard ratio for this comparison is listed above. Listed below is the number of patients at risk (number of patients alive) per interval, after stratification based on CNTNAP2 expression; in the first interval, number at risk is number of patients alive; in each subsequent interval, number at risk is the number at risk less those who have expired or are censored. 


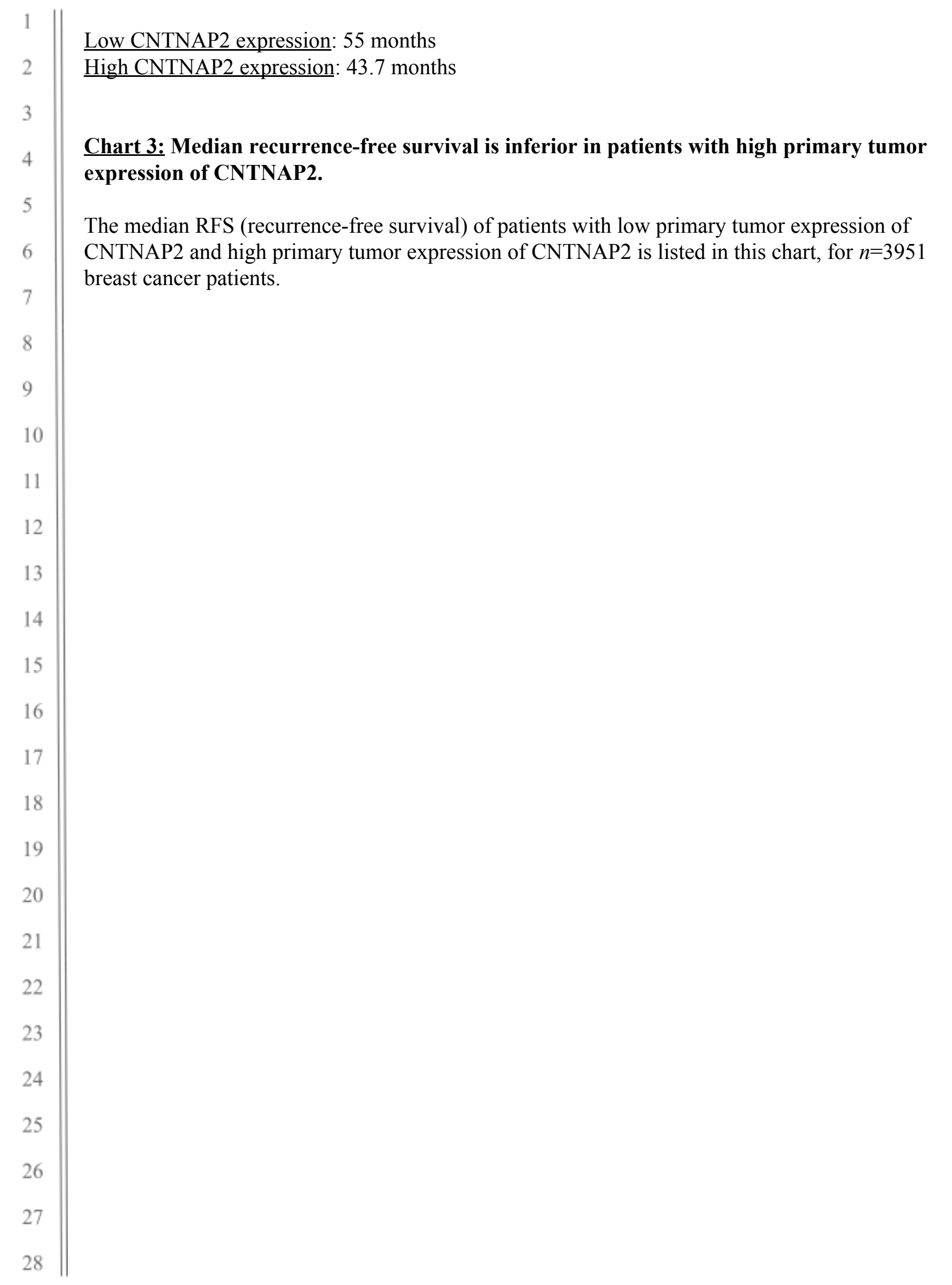

